\title{
Optimal Design and Scheduling of Active Distribution Network with Penetration of PV/Wind/BESS Energy Systems Considering the Load Side Management
}

\author{
Mohammadhosein Kalantari*
}

Department of Power Electric, Ardabil Branch, Islamic Azad University, Ardabil, Iran.

\section{*Corresponding Author: \\ ×Kalantarim@gmail.com}

Received: 15 May, 2021

Accepted: 30 June, 2021

Published: 30 July, 2021

\begin{abstract}
Increasing the penetration of renewable energy sources (RES) in energy systems has had an impressive impact on the design and scheduling of future energy networks and the transition from traditional networks. Sizing and placement of these resources has important technical and economic impacts on the network. However, utilization of these resources in Active Distribution Network (ADN) has several advantages and so, the undesirable effects of these resources on ADN need to be investigated and improved. In this paper, a hybrid ADN includes wind/ PV/ESS, which has been located in 33 IEEE standard bus, is investigated. Optimal energy management and sizing of the RES and ESS are the purposes. Demand Response (DR) is another good option in active networks for regulating production and demand. In this paper, an incentive-based DR program is used. However, this method has uncertainty because it is dependent on customer consumption patterns and the use of inappropriate incentives will not be able to stimulate customers to reduce their consumption at peak times. The optimization problem, which is formulated as optimal programming, is solved to calculate size and placement of each RES as well as ESS condition considering with power losses, voltage profile and cost optimization. The results show the effectiveness of energy management and cost reduction in the studied grid.
\end{abstract}

Keywords: Optimal sizing and placement, Active distribution network, Demand response, $\mathrm{PV} / \mathrm{W}$ ind/BESS

\section{Introduction}

Today, energy plays an important role in the economic, social and industrial development of countries. In general, today energy is supplied through renewable resources and non-renewable resources. From among all types of energy carriers, electricity, though rather expensive, is the cleanest and, one may say, the most important one. On the other hand, fossil fuels as a part of non-renewable resources have a serious impact on the environment as noted by climatologists across the globe. It takes money to extract them from the ground, process them for use and transport them to the end consumer. In addition, the shortage of these resources has raised their prices. But as greater energy consumption means greater energy production, and greater production means more energy resources and more costs, the world finds itself in dire need of some major energy consumption modifications $[1,2]$. Hence more renewable energy sources are needed in future energy systems. By increasing the penetration of renewable energy in the power grid, electricity networks are in the era of major transition from stable passive distribution networks with unidirectional electricity transportation to active distribution networks with bidirectional electricity transportation.

Reference [3] provides a complete overview of the issues related to energy storage resources in active networks. In this paper, location, measurement, economic and social effects, energy security, planning 
and implementation of energy storage resources in the main networks have been investigated. In article [4], the sizing and placement of battery power systems and wind turbines with the aim of reducing cost and system losses are presented. Different strategies for losses reduction and cost optimization have been investigated. The results indicate that the participation of the cost function in the objective function significantly changes the results of the placement and sizing of wind turbines and energy storage resources. In [5], the optimal location of energy storage systems in active distribution networks has been accomplished using the bee colony algorithm. Simulation was performed using DIgSILENT and the results of honey colony optimization algorithm were compared with PSO algorithm. The results indicate improved voltage profiles, reduced power losses, and improved performance of the active distribution network. In [68], the control of the loads and resources of the DER energy distributor is investigated in order to interact the active distribution network with the electricity market. Due to the flexible control of active networks, they can be introduced into three types of integrated load, energy generator, and also as an additional service to the electricity market. The connection between a grid and a grid is carried out by the MC grid controller or the EMS Energy Management System. Proper design of the system is important for ensuring the stability, reliability and economic operation of the grid. In the reference [9], an overview of advanced methods for modeling the uncertainty in the distribution network design has been done. Advantages and disadvantages of each method are stated. Investigations show that the proper choice of the method is proportional to the type of input variables of the uncertainties and the planning problem. The proper installation of a rooftop photovoltaic system in active distribution networks can improve the voltage profile, reduce energy losses, and increase reliability. In reference [10], the location, measurement and charge / discharge of the daily energy storage system in active distribution networks is investigated by integrating the photovoltaic system. Simulations have been done in MATLAB and DIGSILENT software. The results show that the overvoltage and energy losses are reduced by using the stated method, environmental pollution decreases and economic profitability increases. Using Partitioning strategy to solve the voltage fluctuation problem, decentralized modeling for controlling the voltage of renewable resources, and applying the sensitivity matrices of some of the topics discussed in reference [11]. The results of simulations in 34 and 69 distribution test system distribution systems show the effective performance of the proposed method. In the reference [12], two centralized and decentralized control methods are provided for controlling active networks connected to the main network in the electricity market. In the first method, the local DER controller receives commands from the MC, and in the decentralized model, the local DER controller is used to maximize the decision function's target function. The purpose of this paper is to maximize the profit of the grid by participating in the electricity market, which is the MC interface between the grid and the market operator. A new voltage control method for radial active distribution networks is proposed to regulate the effective voltage with high penetration of distributed generation in [13]. This method has low computational load and reduces active power losses. Reference [14] proposed an economic strategy to reduce costs based on a 24-hour forecast that the EMS predicts using the neural network to generate output and load. In addition, optimal load distribution has been calculated based on economic analysis using the metaoptimization method. The greater penetration of renewable energy sources results in reverse power flows, voltage, and critical issues in distribution networks. In the reference [15], the problem of planning a medium voltage network with the influence of renewable resources has been investigated. In this paper, the optimal location of the energy storage resources and the sub-loader tap changers are simultaneously implemented. The use of a secondorder programming model and a nonlinear model of a sub-chip loader transformer yield significant results. In [16], a review of optimal locating strategies and energy storage systems sizing in active distribution networks has been carried out. Technologies and benefits of using energy storage systems, recent methods for optimal allocation, and control strategies are investigated. In [17], long-term planning has been done to optimize the design of distributed generations and installed batteries and short-term planning optimizes the performance of the generation units of production and batteries. The reactive power for DGs and batteries has been considered and the battery charge depth has been optimized as a design variable for the batteries. A probabilistic planning model with uncertainty and the development of a multi-objective optimization method for the proposed model in active distribution networks are given in [18]. In [19], the integration of the ESS is done to improve the network performance is based on active network management methods. In this paper, the level of ESS and its placement to reduce constraints and improve utilization of active distribution networks have been investigated. Considering the advantages of using power storage systems in distribution networks, a statistical programming method for voltage regulation with a high penetration level of renewable resources has been investigated in paper [20].

In this paper the placement and sizing of the wind and solar resources along with the battery energy storage in the 33-IEEE standard is investigated to reduce the investment and operational costs of the active 
distribution network. The outputs of the voltage profile and overall cost of the system are indicated the proper performance of the proposed strategy despite all strict constraints such as geometric constraints, voltage stability index, voltage deviation, capacity constraints of RES along with demand response management constraints.

\section{Optimal Sizing of WT and PV}

Wind energy and solar energy are used extensively in distribution networks among various sources of distributed generation. Modeling and predicting precisely the wind speed and solar radiation is very complicated. In this paper, firstly, the wind speed is predicted based on the Weibull method, and then its output power is calculated. For these calculations, the hourly values of wind speed and radiation are considered. The meteorological statistical data is used to model wind and solar power systems, which is explained in the results and discussion section.

\section{PV System Modeling}

The output power of the photovoltaic system is a function of solar radiation, panel area, temperature and solar absorption capacity of the solar panel, which is expressed by:

$$
\begin{array}{ll}
\Pi=\Pi \times{ }^{\Gamma} \times[1+K((T+(0.0256 \times \Gamma))-T)] & \\
\pi \varpi-o v \tau N-\pi \varpi \Gamma^{\tau \alpha \mu \beta} & \rho \varepsilon \phi
\end{array}
$$

where, $P_{p v-o u t}$ is the output power, $G$ is reference irradiation $\left(1000 \mathrm{~W} / \mathrm{m}^{2}\right), T_{r e}$ is reference ambient temperature $\left(25^{\circ} \mathrm{C}\right)$.

\section{Wind Turbine (WT) Modeling}

A wind turbine extracts the energy from moving air masses to convert it into electric energy. Wind power acts on the rotor blades into torque and subsequently the rotational energy is used within a generator for electricity production. The power generated from the wind turbine can be expressed in the following formula $[21,22]$.

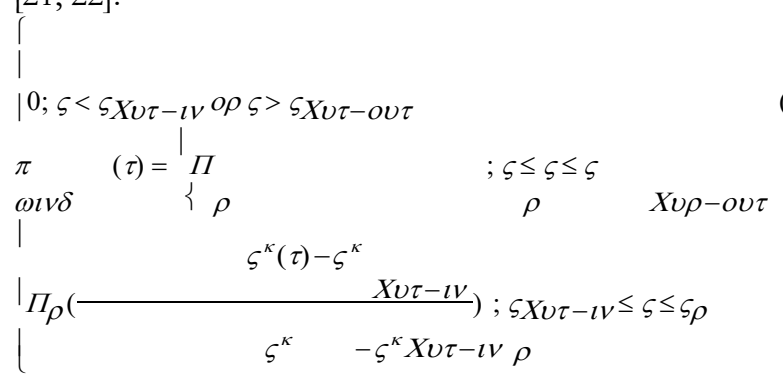

where $P_{r}$ is the rated power of the WT, $V_{C u t-i n}$ is the cut-in wind speed, $V_{r}$ is the rated wind speed, $V_{C u t-}$ out is the cut-out wind speed and $k$ is the Weibull shape parameter. If the number of wind turbines is $N_{\text {wind }}$, the overall produced power is $P_{\text {wind }}(t) \square N_{\text {wind }} \square$ pwind $(t)$

\section{Problem Formulations}

The classic problem of electric networks is the economic load dispatch of generating systems to achieve minimum operating cost. In addition, there is a need to expand the limited economic optimization problem to incorporate constraints on system operation to ensure the security of the system, thereby preventing the collapse of the system due to unforeseen conditions. With the integration of RES the problem of load dispatch has changed and the objective function and constraints of the problem need to be modified.

Power Loss Reduction: This paper considers the active power loss reduction of the network as objective function, which mathematically can be expressed as:

$$
\phi=\mu \operatorname{lv}\left(\sum \sum_{1}^{N_{\sigma \varepsilon}} \sum^{N \tau} \sum^{l=N \beta-1} \Pi^{\tau}\right)
$$

Voltage Stability Index (VSI): VSI is an indicator which shows the stability of distribution system [23]. Equation (4) represent the mathematical expression for the voltage stability index as an objective function.

$$
\begin{aligned}
& \varsigma \Sigma I \quad=\varsigma^{4}-4(\Pi \times \Xi-\Theta \times P)^{2}-4(\Pi \times P+\Theta \times \Xi) \varsigma^{2}
\end{aligned}
$$

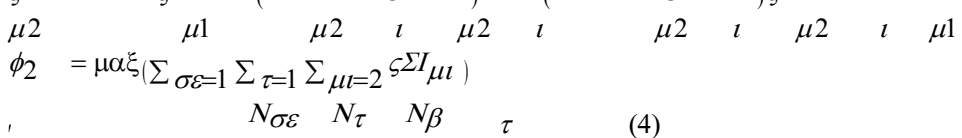

Voltage Deviation: The radial nature of distribution system causes voltage dips in heavy load and remote areas. The mathematical index can be formulated as in the following equation (5):

$$
\left.\phi=\mu \imath v_{\left(\sum\right.} \sum_{3}^{N_{\sigma \varepsilon}} \sum_{\tau}^{N_{\tau}} \sum^{N_{\beta}} \quad 1-\rho \varepsilon \alpha \lambda(\varsigma)^{\tau}\right)
$$

\section{Network Constraints}

Power Balance: According to Kirchhoff law or system power balance, the generated power and consumption power should be equal in order that the power system to be stable.

$$
\begin{aligned}
& N_{\Omega T}(\tau) \times \Pi_{\Omega T}(\tau)+N_{\pi \varpi}(\tau) \times \Pi_{\pi \varpi}(\tau)+\Pi_{\delta}(\tau)=\Pi_{\lambda o \alpha \delta} \\
& (\tau)+\Pi_{\chi}(\tau)(6)
\end{aligned}
$$

where $N W T(t), N P V(t)$ are the number of WTs and the number of PVs respectively. PWT(t), PPV (t) are the rated power of WT and PVs. $P_{c}(t)$ and $P_{d}(t)$ are the charge and discharge power and Pload (t) is the load power. 
Position of RES: Bus 1 is the substation or slack bus, so the position of the RES should not be used at bus 1:

$$
2 \leq P E \Sigma_{\pi \circ \sigma \imath \imath \iota \mathrm{v}} \leq v_{\beta v \sigma \varepsilon \sigma \quad(7)}
$$

Voltage Magnitudes:

$$
\varsigma^{\tau} \leq \varsigma \leq \varsigma, \quad \mu k
$$

Constraints of RES

To solve the optimization problem, the following constraints must be considered for RES.

Kirchhoff law or system power balance: The generated power and consumption power should be equal in order that the power system to be stable.

$N_{\Omega T}(\tau) \times \Pi_{\Omega T}(\tau)+N_{\pi \varpi}(\tau) \times \Pi_{\pi \varpi}(\tau)+\Pi_{\delta}(\tau)=\Pi_{\lambda \circ \alpha}$ $\delta(\tau)+\Pi_{\chi}(\tau)$

where $N W T(t), N P V(t)$ are the number of WTs and the number of PVs respectively. PWT (t), PPV (t) are the rated power of WT and PVs. $P_{c}(t)$ and $P_{d}(t)$ are the charge and discharge power and Pload $(t)$ is the load power.

Energy storage is used for balancing generation and consumption power. The new state of charge for the battery bank is given as follow:

$\Sigma O X(\tau)=\Sigma O X(\tau-1)+\Pi_{\chi}(\tau) \eta_{\chi}-\Pi_{\delta}(\tau) / \eta_{\delta}$

In the above relations, $S O C(t)$ and $S O C(t-1)$ are the battery bank state of charge at the times $t$ and $t-1$, respectively, $\eta_{c}$ is the efficiency of the charging batteries and $\eta_{d}$ is the efficiency of the discharging batteries. The initial state of charge at the beginning of the simulation is considered as follow:

$$
\Sigma O X(0)=\Sigma O X_{\beta} \times E_{\beta \mu \alpha}
$$

$S O C_{b}$ is 0.2 and Ebmax is maximum capacity of the batteries, $S O C(0)$ is the initial state of charge.

Energy storage constrains: The constraint in below equations is imposed for charge and discharge to prevent a reduction in the useful life of each battery.

$$
\begin{array}{ll}
\Sigma O X(\tau)=N(\tau) \times E & \\
\beta & \beta \mu \alpha \xi \\
\Sigma O X(\tau)=N(\tau) \times E & \\
\beta & \beta \mu \mathrm{v}
\end{array}
$$

where $\mathrm{Nb}(t)$ is the number of ESS, Ebmax and Ebmin is the maximum and minimum capacity of the ESS.

To prevent simultaneous charge and discharge of the ESS, these constrains are considered which $M$ is the positive large number that must be greater than the capacity of the batteries.

$$
\Pi_{\chi}(\tau) \leq M \times \imath \varepsilon \varepsilon \chi(\tau)
$$

$$
\begin{aligned}
& \Pi(\tau) \leq M \times \imath \varepsilon \varepsilon \delta(\tau) \\
& \delta \\
& \imath \varepsilon \varepsilon \chi(\tau)+\imath \varepsilon \varepsilon \delta(\tau) \leq 1 \quad \imath \varepsilon \varepsilon \chi \alpha v \delta \imath \varepsilon \varepsilon \delta \in\{0,1\}
\end{aligned}
$$

where, ieec and ieed are the charge and discharge status of the battery bank at the time $t$, respectively.

Economic constraints: Cost limitation of PV panels, wind turbines and energy storage is considered, which the installation cost of components should not exceed assuming maximum available budget:

$X_{\mathrm{\imath v} \tau_{-} \pi \varpi} \times N_{\pi \varpi}+X_{\mathrm{\imath v} \tau_{-} \omega \imath v \delta} \times N_{\omega \imath v \delta}+X_{\mathrm{\imath v} \tau_{-} \beta \alpha \tau} \times N_{\beta \alpha \tau} \leq X_{\beta \gamma}(17)$

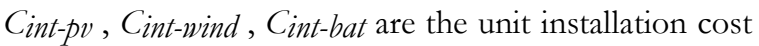
of a PV panel, WT and ESS. Cbg is the maximum available budget.

Geometric constraints: The limited available ground area for the installation of the wind turbines and PV is considered with respect to the wind and PV energy production, $A b$ and $S b$ are the basic ground area occupied by a wind turbine and PV, respectively; $A_{\max }$ and $S_{\max }$ are the available area for wind turbine and $\mathrm{PV}$, respectively:

$$
\begin{aligned}
& N_{\omega \imath \nu \delta} \times A_{\beta} \leq A_{\mu \alpha \xi} \\
& N_{\Pi_{\zeta} \times \Sigma_{\beta} \leq \Sigma_{\mu \alpha \xi}}
\end{aligned}
$$

Capacity constraints of MGs:

$$
\begin{aligned}
& N^{\mu \mathrm{\imath \nu}} \leq N^{\kappa} \leq N^{\mu \alpha \xi} \\
& \Pi_{\varsigma} \quad \Pi_{\varsigma} \quad \Pi_{\varsigma} \\
& N^{\mu \mathrm{\imath \nu}} \leq N^{\kappa} \leq N^{\mu \alpha \xi} \\
& \Omega T \quad \Omega T \quad \Omega T
\end{aligned}
$$

$\mathrm{N} \mathrm{N} \mathrm{N}$ and $\mathrm{N}$ are the minimum and maximum number of the WTs and PVs which calculated by the following equations:

$$
\begin{aligned}
& \alpha \sum^{24} \Pi(\tau) \\
& N^{\mu \mathrm{\nu} \nu}=\quad \tau=1 \Lambda
\end{aligned}
$$

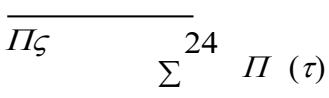

$$
\begin{aligned}
& \tau=1 \Pi \varsigma \\
& \beta \sum^{24} \Pi(\tau) \\
& N^{\mu \alpha \xi}=\quad \tau=1 \Lambda \\
& \Pi_{\varsigma} \quad \Sigma^{24} \Pi(\tau) \\
& \tau=1 \Pi \varsigma \\
& \gamma \sum^{24} \Pi(\tau) \\
& N^{\mu \mathrm{\imath \nu}}=\quad \tau=1 \Lambda \\
& \overline{\Omega T \quad \Sigma^{24} \Pi(\tau)}
\end{aligned}
$$


$\tau=1 \quad \Omega T$

$$
\begin{aligned}
& \lambda \sum^{24} \Pi(\tau) \\
& N^{\mu \alpha \xi}=\sum_{\tau T}^{24} \sum^{24} \Pi(\tau) \\
& \tau=1 \quad \Omega T
\end{aligned}
$$

where $a, \beta, \gamma$, and $\lambda$ are the scaling factors.

\section{Demand Response Management}

The electric power system of today requires a good balance of demand and supply, a smooth and stable delivery and environmentally friendly produced electricity to avoid climate change. In addition, electricity consumption should be reduced and/or shifted to eliminate environmental emissions, decrease costs, ensure safe electricity supply and enable more integration of renewable intermittencies. Security of supply and a rising demand are two factors impelling demand side management and Demand Response is the method for reducing or shifting electricity consumption at the demand side and there are many varieties within the concept. According to IEEE expression, Demand Side Management is a portfolio of measures to improve the energy system at the side of consumption. It ranges from improving energy efficiency by using better materials, over smart energy tariffs with incentives for certain consumption patterns, up to sophisticated realtime control of distributed energy resources" [24]. There are different models for demand response programs: direct load control and price response control are two defined options. Load control enables customers to sign a contract about the reduction that could be controlled automatically without any further action from them. Price response control requires a higher degree of customer participation but that degree depends on the model. This demand elasticity (E) pertaining to electricity price (EP) [3] is defined as:

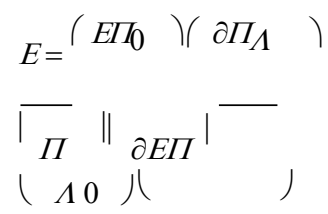

Where $E P_{0}$ and $P_{L 0}$ are initial electricity price and load demand respectively, $\partial E P$ and $\partial P_{L}$ describe the change in electricity price and load demand from their initial values respectively. Customer behavior is characterized according to the load variation with the change in the electricity price. There are certain inflexible loads which cannot shift from one period to another with the price variation and are sensitive to single period only. These loads are termed as self-elasticity. Furthermore, some elastic loads that can vary from peak hours to low load periods having sensitivity to multi-period can be defined as cross elasticity. Consequently, customer behavior for $24 \mathrm{~h}$ can be epitomized by price elasticity matrix (PEM) which is a $24 \times 24$ matrix with self- elasticity coefficients as diagonal elements and cross elasticity coefficients as off-diagonal elements [16]. In DRP, the participating customers change their load demand according to (27).

$$
\begin{aligned}
& (E \Pi-E \Pi+A) \\
& \Pi=\Pi \quad \varepsilon \xi \pi_{\mid} E \quad(\eta) \quad 0(\eta) \quad(\eta)
\end{aligned}
$$

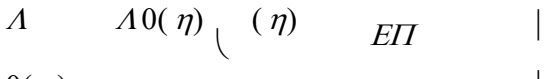

$$
\begin{aligned}
& 0(\eta)
\end{aligned}
$$

where $A$ is the incentive value and $E P(b)$ is the electricity price at $h$-th hour.

\section{Standard PSO Algorithm}

PSO starts with a population of random solutions "particles" in a $D$-dimension space. The $i$ th particle is represented by $\mathrm{X}_{i}=(\mathrm{x} i 1, \mathrm{x} i 2, \ldots, \mathrm{x} i D)$. Each particle keeps track of its coordinates in hyperspace, which are associated with the fittest solution it has achieved so far. The value of the fitness for particle $i$ (pbest) is also stored as $P_{i}=\left(p_{i 1}, p_{i 2}, \ldots, p_{i} D\right)$. The global version of the PSO keeps track of the overall best value (gbest), and its location, obtained thus far by any particle in the population. PSO consists of, at each step, changing the velocity of each particle toward its pbest and gbest according to Eq. (28). The velocity of particle $i$ is represented as $V i=(v i 1, v i 2, \ldots, v i D)$. Acceleration is weighted by a random term, with separate random numbers being generated for acceleration toward pbest and gbest. The position of the $i$ th particle is then updated according to (29) $[25,26]$.

$\varpi_{l} \delta=\omega \varpi_{l} \delta+\chi 1 \rho \alpha v \delta()\left(\Pi_{l} \delta-\xi_{l} \delta\right)+\chi 2 \rho \alpha v \delta()\left(\Pi_{\gamma}(28)\right.$

$\left.\delta-\xi_{\imath} \delta\right)$

$\xi_{l \delta}=\xi_{l \delta}+\chi \varpi_{l \delta}$

where, $P_{i d}$ and $P_{g d}$ are pbest and gbest. One evident advantage of PSO is the ease of implementation.

Modified Particle Swarm Optimization Algorithm

In standard PSO, because the particle has the ability to know the best position of the group particles have been searched, we need one particle to find the global best position rather than all particles to find it, and other particles should search more domains to make sure the best position is global best position not the local one. Based on these ideas, we propose some modifications with the standard PSO algorithm. 
The PSO algorithm is further improved through using reduction in the number of iterations [24, 21]. The performance of this modified algorithm depends on the method of tuning the inertia weight. In this work, a linearly-decreasing time-dependent inertia weight proposed in [22] has been implemented, according to the following update law:

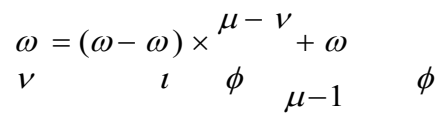

where $\omega_{i}$ is initial weight, $\omega_{f}$ is final weight, $m$ is maximum iteration value and $n$ is variable iteration index.

Note here that the inertia weight $\omega$ plays an important role in the convergence of the PSO algorithm to the global optimal solution and hence has an influence on the time taken for a simulation run. Recall here that the weight factor is used to control the influence of the previous history of the particle velocities on both the current velocity and the local and global exploration capabilities of the PSO algorithm. It thus follows that the reason for using a linearly-decreasing-in-time inertia weight parameter $\omega$ is that larger values of $\omega$ tend to be used at the start of the search to enable the PSO algorithm to explore globally the solution space, whereas smaller values of $\omega$ are used toward the end of the search to enable the PSO algorithm to explore locally around the global optimum before finally homing in onto it.

Recent works in [21] and [22] indicate that the use of a "constriction factor" may be necessary to insure convergence of the PSO. A simplified method of incorporating a constriction factor is represented in [21]:

$\varpi \iota \delta=K \times[\varpi \iota \delta+\chi 1 \rho \alpha v \delta()(\Pi \imath \delta-\xi \imath \delta)+\chi 2 \rho \alpha v \delta(\Pi \gamma \delta-\xi \imath \delta)]$ where $K$ is a function of $c_{1}$ and $c_{2}$ as illustrated by the following equation (31). a time-decreasing inertia weight, which leads to a

$$
\begin{array}{l|l}
K= & \kappa \\
2-\varphi-\varphi^{2}-4 \varphi & (32)
\end{array}
$$

where $k=2, \varphi=\chi 1+\chi 2, \alpha v \delta \varphi>4$. In [25], the performance of PSO using an inertia weight was compared with the PSO performance using a constriction factor. It was concluded that the best approach is to use a constriction factor while limiting the maximum velocity $v_{\max }$ to the dynamic range of the variable $x_{\max }$ in each dimension. It was also shown in that this approach provides a performance superior to any similar technique reported in the literature.

\section{Simulation Results and Discussion}

The simulations are performed in two stages. In the first step, considering the region's solar and wind power potential and location and cost constraints, the number and capacity needed to supply roughly $25 \%$ of demand load power by renewable sources and BESS is determined. In the second step, the optimal placement of renewable sources and BESS is done according to the voltage profile index, voltage stability index and system power losses.

Step 1: In this study, weather and geographical information of Queensland-Australia is used to investigate the scheduling of a hybrid system. This area is desirable to extend the WT system, because it receives highest amount of wind power throughout the year. Also, solar irradiation potential of there is suitable for active distribution networks. profile of typical daily load is shown in Fig. 1. Techno-economical parameters $8 \mathrm{f}$ microgrid components are tabulated in Table 1 and the PEM is shown in Table 2. Fig. 2 shows the wind speed in Queensland-Australia city during a year.

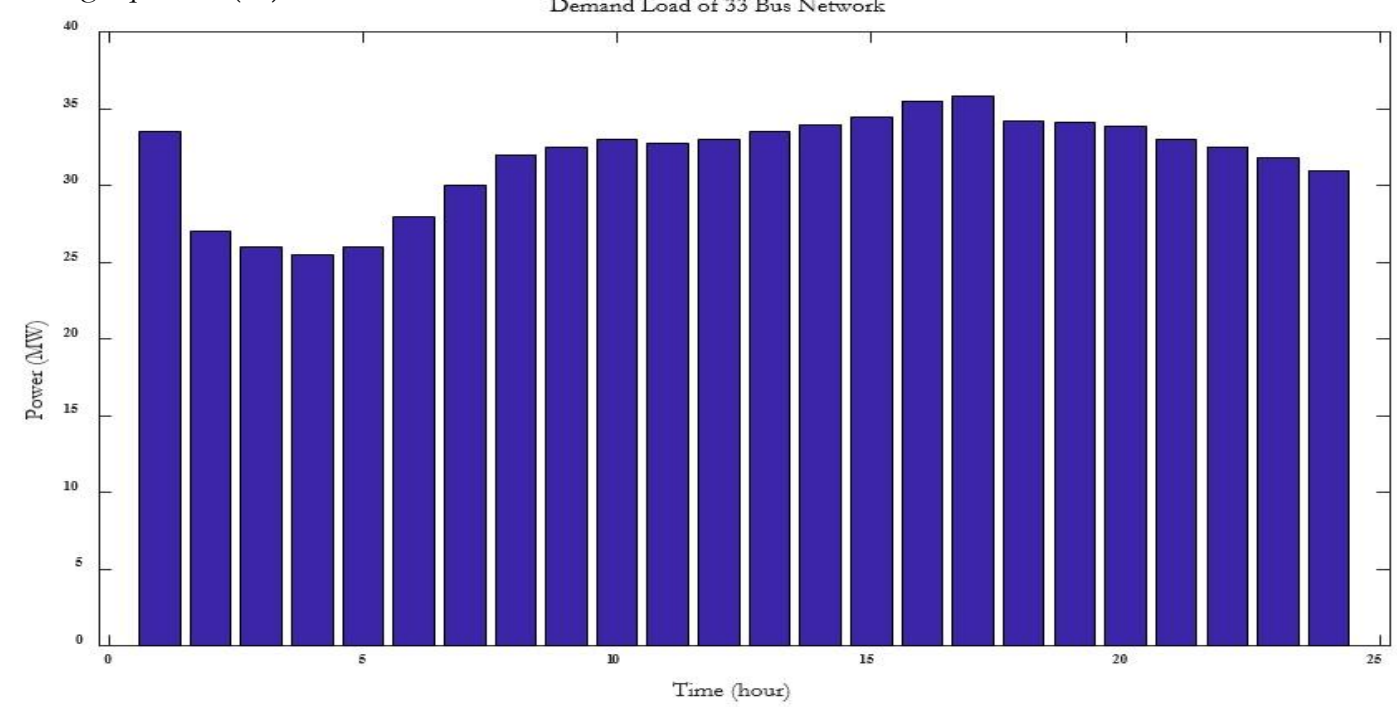




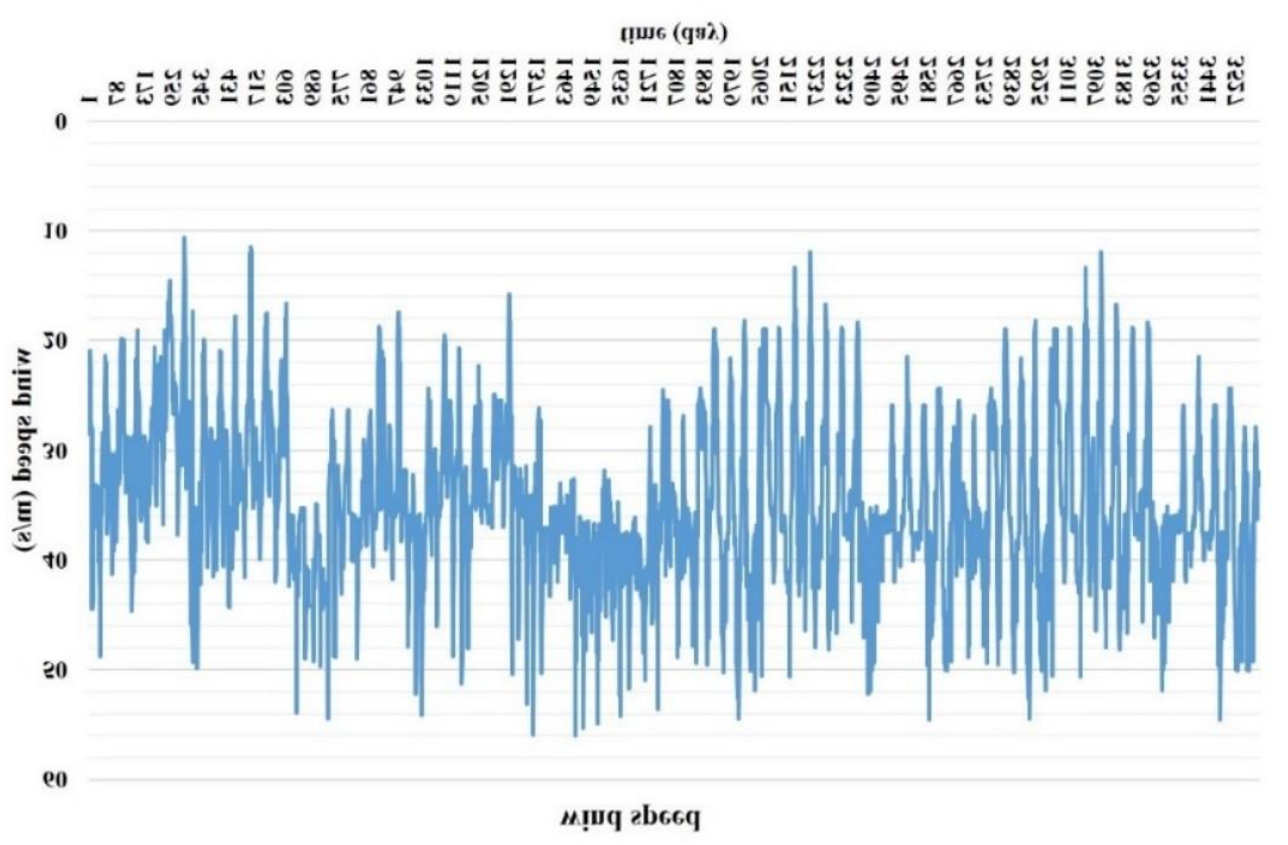

Figure 1. Daily load profile

Figure 2. Mean wind speed for one year

Table 1

The grid data

\begin{tabular}{|c|c|c|c|}
\hline \multicolumn{2}{|l|}{ Wind parameters } & \multicolumn{2}{|c|}{ Battery parameters } \\
\hline$v_{\text {cut }-i n}$ & $2.5 \mathrm{~m} / \mathrm{s}$ & Voltage & $12 \mathrm{v}$ \\
\hline$v_{\text {cut_out }}$ & $13 \mathrm{~m} / \mathrm{s}$ & $E_{b \max }$ & $70 \mathrm{kwh}$ \\
\hline Cint-wind & 3200 \$/unit & $E_{b \text { min }}$ & $13.2 \mathrm{kwh}$ \\
\hline$v_{r}$ & $9.5 \mathrm{~m} / \mathrm{s}$ & $\eta^{\text {batt }}$ & $85 \%$ \\
\hline$P_{r}$ & $5 \mathrm{kw}$ & $C C_{\text {bat }}$ & $130 \$ /$ unit \\
\hline$C C_{\text {wind }}$ & $3200 \$$ /unit & Life span & 5 years \\
\hline $\begin{array}{l}O P \& M I \\
\text { wind wind }\end{array}$ & $100 \$ /$ year & dod & 0.8 \\
\hline Life span & 20 years & $\operatorname{soc}_{0}$ & $13.2 \mathrm{kwh}$ \\
\hline Available area for wind & $80 \mathrm{~m}^{2}$ & $\begin{array}{l}O P \& M I \\
\text { bat } \quad \text { bat }\end{array}$ & $5 \$ /$ year \\
\hline PV parameters & & Load parameters & \\
\hline${ }_{P}^{P V N}$ & $1 \mathrm{kw}$ & households & 15 \\
\hline$O P_{P V} \& M I_{P V}$ & $5 \$ /$ year & Budget available & $5000000 \$$ \\
\hline$C C_{p v}$ & $1800 \$$ /unit & $\begin{array}{l}\text { Load average } \\
\text { each house }\end{array}$ & 2.558 \\
\hline Life span & 25 year & $\eta^{i n v}$ & 0.95 \\
\hline$\eta P V$ & 0.85 & $i$ (interest rate) & 0.06 \\
\hline Available area for PV & 1.7 & & \\
\hline Cint-pv & $1800 \$$ /unit & & \\
\hline
\end{tabular}

The MPSO algorithm is applied in order to obtain the best configuration of system and for sizing the components. The objective function is minimization of the total cost. The solution deals with the optimum 
component size of the grid. By considering the results of simulations the minimum number of WT and PV are The impact of the budget and geometric constraints into a microgrid size optimization is studied in two scenarios.

Scenario 1: cost minimization with considering budget and geometric constraints.

Scenario 2: cost minimization by considering all constraints and EDRP.

The optimization is performed separately for one sample day each in season of the year, for considering both the load and seasonality variability of wind speed and solar radiation. In this scheduling problem, because of choosing 4 days which represent four seasons in a year, there is no continuity between days. So, energy considered 10 and 50 and the maximum number are 65 and 170 respectively.

interchange between ESS and load-generation system must be settled in each day. The Fig. 3 nd Fig. 4 show the mean wind power generation in each season and mean PV power generation in each season, respectivelly. The results show that MPSO algorithm provides optimum wind, PV and ESS ratings. The best-founded solution presented in the Table 3 and 4 . The associated costs of the ADN are also presented. The optimization results for the sample days are reported in Fig. 5 and Fig. 6 for in scenario 1 and scenario 2, respectively. Fig. 6 shows the flexibility of loads in the demand response program that has shifted from peak hours to off-peak times.

Table 2

Price elasticity matrix.

\begin{tabular}{lccccc}
\hline & Low & Off-peak & peak & Off-peak & peak \\
\hline Low & -0.08 & 0.03 & 0.034 & 0.03 & 0.034 \\
Off-peak & 0.03 & -0.11 & 0.04 & 0.03 & 0.04 \\
peak & 0.034 & 0.04 & -0.19 & 0.04 & 0.01 \\
Off-peak & 0.03 & 0.03 & 0.04 & -0.11 & 0.04 \\
peak & 0.034 & 0.04 & 0.01 & 0.04 & -0.19 \\
\hline
\end{tabular}

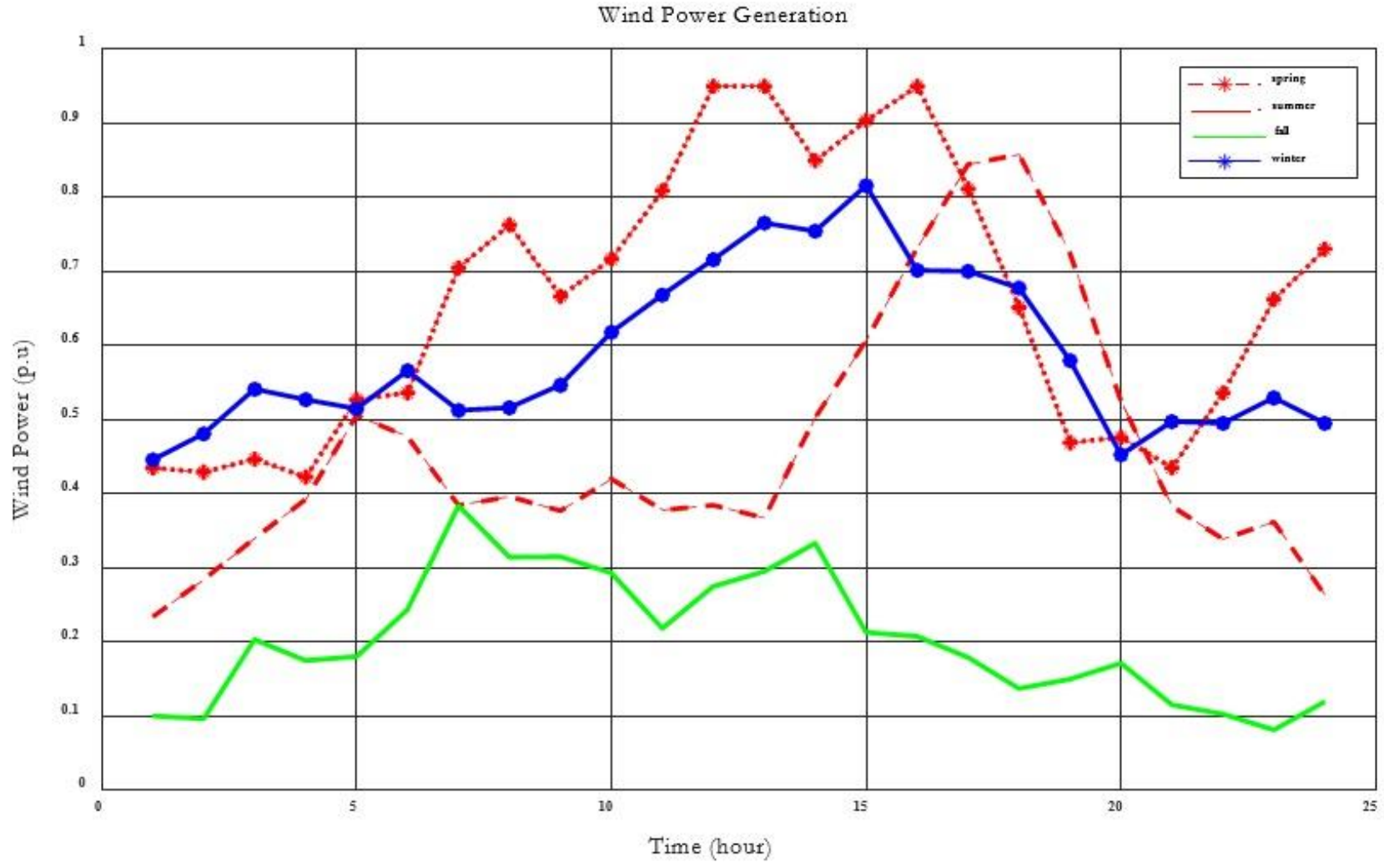

Figure 3. mean wind power generation in each season. 


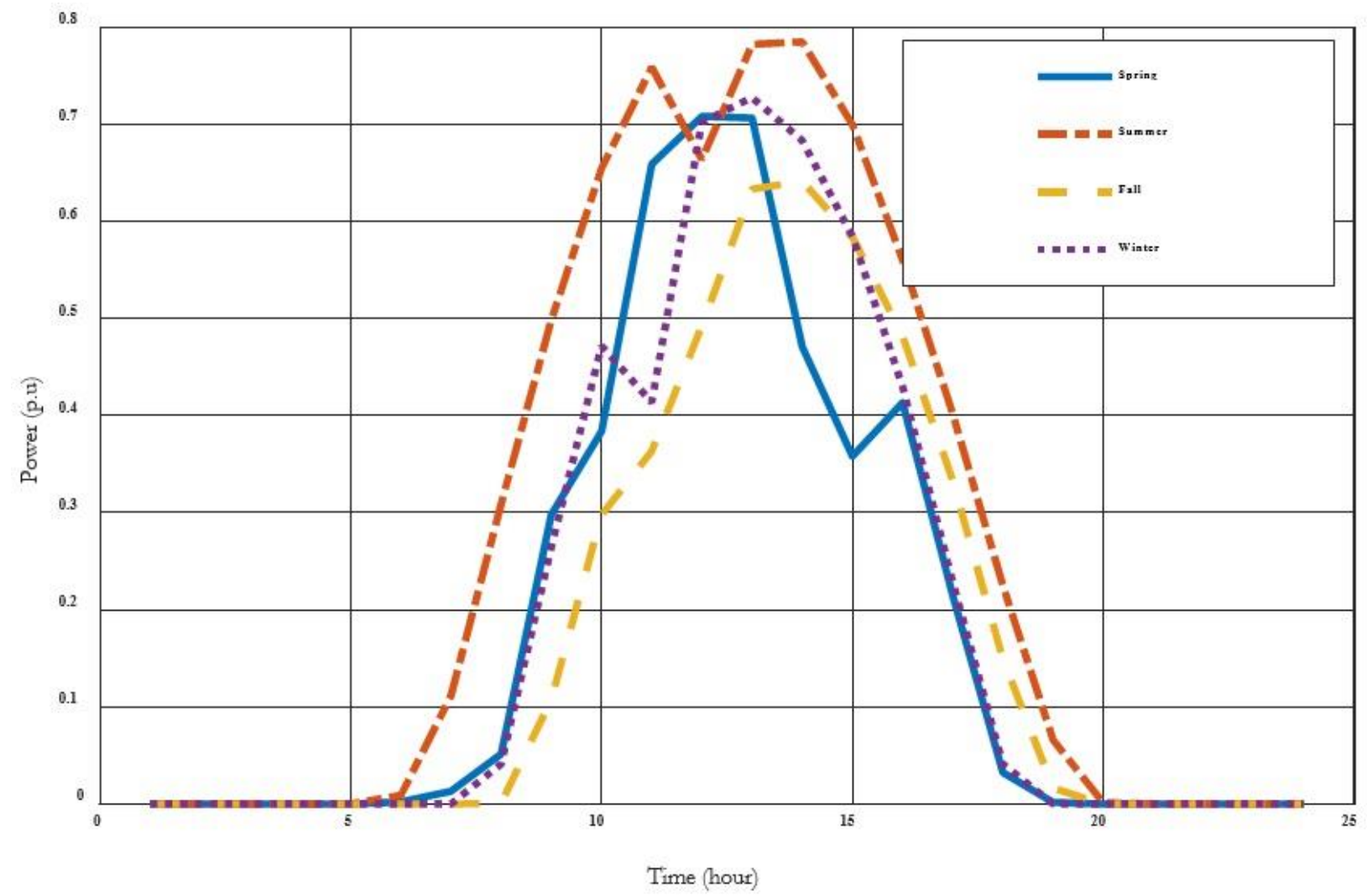

Figure 4. Mean PV power generation in each season

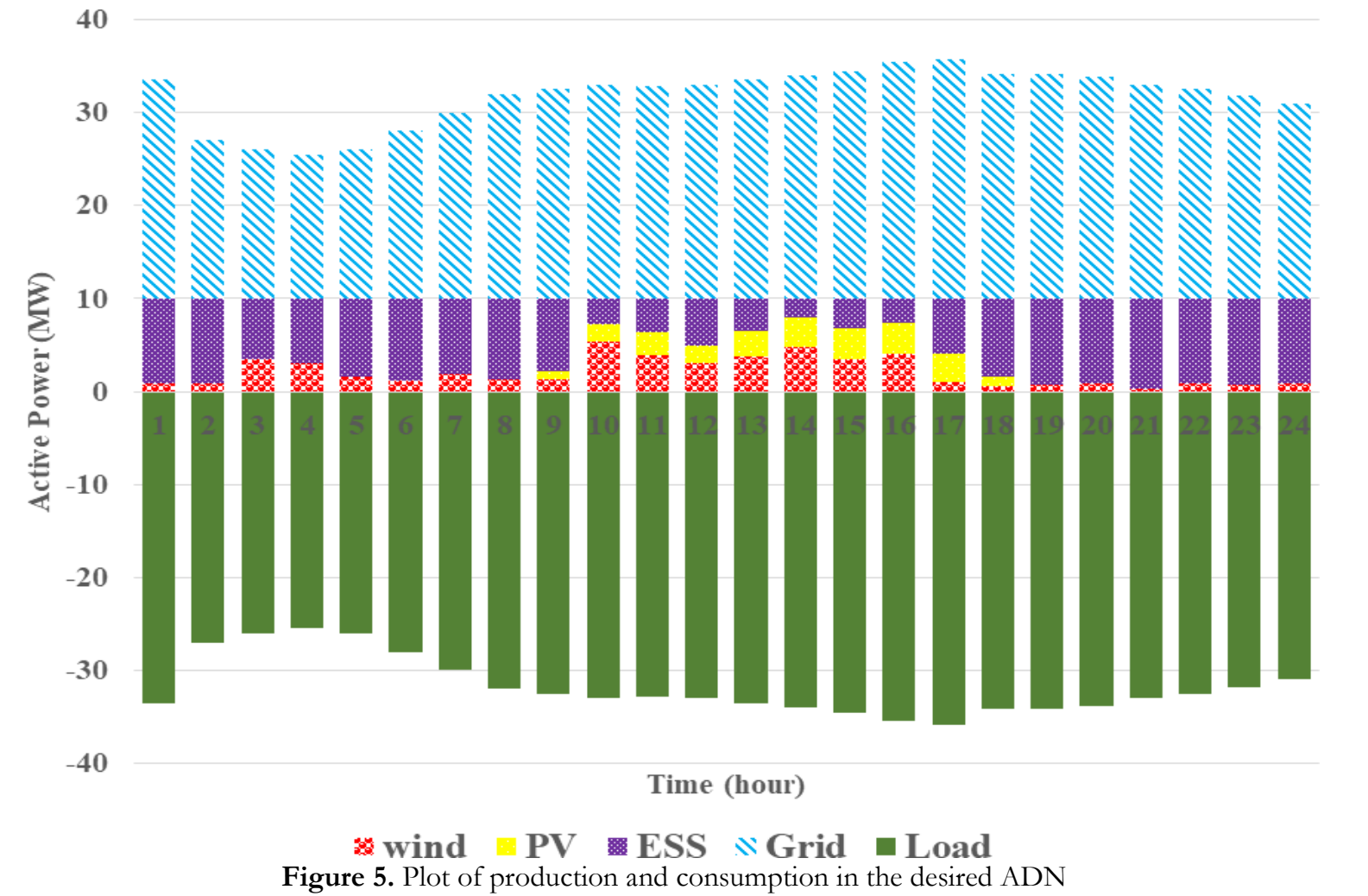




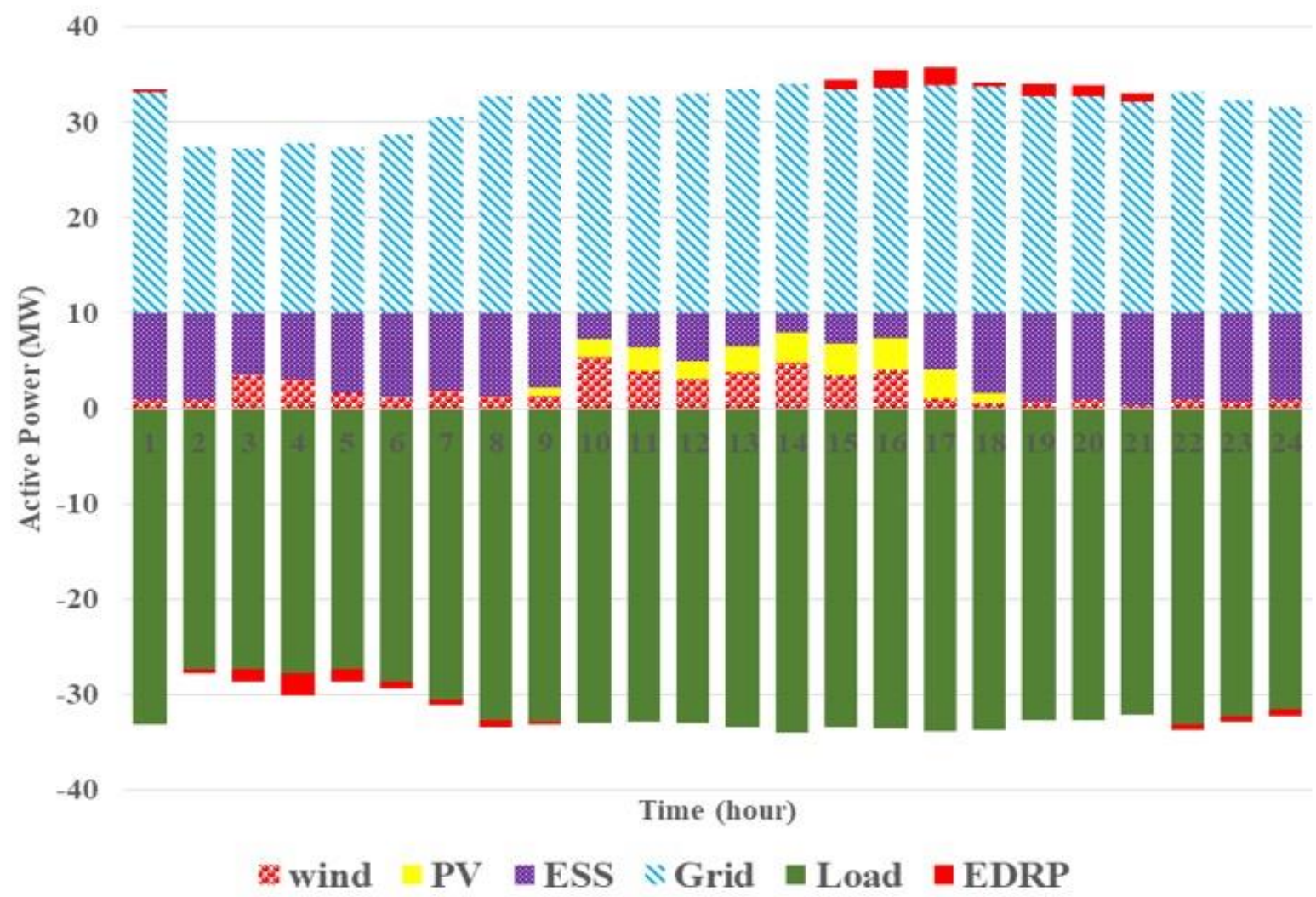

Figure 6. Plot of production and consumption in the desired ADN wit DRP

Table 3

Optimal component size and total cost for first scenario.

\begin{tabular}{lcccc}
\hline & \multicolumn{4}{c}{ Scenario 1 } \\
\cline { 2 - 5 } & spring & summer & Fall & winter \\
\hline Number of WT & 20 & 28 & 65 & 22 \\
Number of PV & 170 & 170 & 228 & 170 \\
BESS & 1 & 2 & 2 & 1 \\
Cost $\left(\times 10^{3} \$\right)$ & 1001.795 & 996.678 & 1013.790 & 1005.253 \\
\hline
\end{tabular}

Table 4

Optimal component size and total cost for second scenario.

\begin{tabular}{lcccc}
\hline & \multicolumn{4}{c}{ Scenario 2 } \\
\cline { 2 - 5 } & spring & summer & Fall & winter \\
\hline Number of WT & 20 & 28 & 64 & 22 \\
Number of PV & 147 & 147 & 147 & 147 \\
BESS & 1 & 1 & 2 & 2 \\
Cost $\left(\times 10^{3} \$\right)$ & 1000.474 & 994.077 & 1012.824 & 1001.763 \\
\hline
\end{tabular}

Step 2: In the second step of the simulation, the optimal locating of the sources is done according to the system losses and cost and voltage stability. At this step, five combination cases of PV, WT, and batteries are considered for optimal placement in buses. The results of the simulations are presented in Table 5. The optimal number and location of PV, WT and BESS along with voltage stability index are presented in this table. The buses voltage profiles for 5 case studies are shown in Fig. 7. 


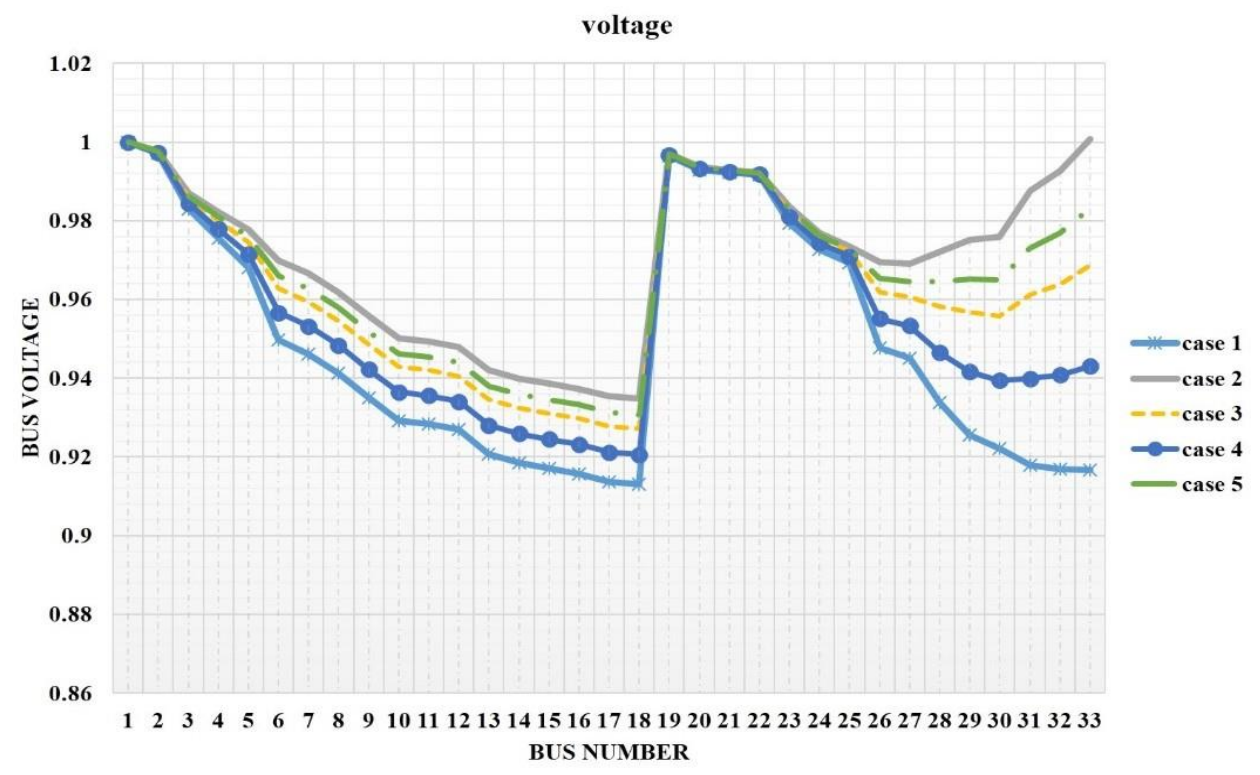

Figure 7. voltage profiles for 5 case study.

Table 5

Placement, power losses and cost of 5 case studies.

\begin{tabular}{lcccccc}
\hline \multirow{2}{*}{$\begin{array}{l}\text { Case } \\
\text { Studies }\end{array}$} & $\begin{array}{c}\text { No. of } \\
\text { WT }\end{array}$ & Locations & $\begin{array}{c}\text { No. of } \\
\text { PV }\end{array}$ & Locations & $\begin{array}{c}\text { No. of } \\
\text { BESS }\end{array}$ & Locations \\
\hline Case 1 & 0 & --- & 0 & --- & 0 & --- \\
Case 2 & 2 & 18,29 & 2 & 7,27 & 2 & 24,30 \\
Case 3 & 3 & $18,29,30$ & 2 & 10,27 & 2 & 24,30 \\
Case 4 & 3 & $18,29,30$ & 3 & $13,27,30$ & 2 & 28,30 \\
Case 5 & 3 & $18,29,30$ & 3 & $13,24,30$ & 3 & $27,28,30$ \\
\hline
\end{tabular}

\section{Conclusion}

The penetration of renewable energy sources in ADN with its advantages in the field of environmental pollution and reduction of dependence on fossil fuel sources offers important challenges for energy networks. These issues need to be addressed by appropriately locating and sizing of RES along with BESS due to their alternate nature of solar and wind energy resources. Meanwhile, with the development of information technology infrastructures and their applications in power grids, the issue of load management also plays an important role in the generation scheduling. In this paper, the problem of optimal placement and sizing of the wind and solar resources with battery energy storage in the 33-IEEE standard bus is implemented based on a flexible demand response program with the MPSO Algorithm. The outputs of the voltage profile and overall cost of the system are indicated the proper performance of the proposed strategy despite all strict constraints such as geometric constraints, voltage stability index, voltage deviation, capacity constraints of RES and demand response management constraints.

\section{References}

1. Babacan O, Torre W, Kleissl J. Siting and sizing of distributed energy storage to mitigate voltage impact by solar PV in distribution systems. Solar Energ. 2017; 146: 199-208.

2. Junaid Khana M, Kumar Yadavb A, Mathew L. Techno economic feasibility analysis of different combinations of PV-WindDiesel-Battery hybrid system for telecommunication applications in different cities of Punjab, India. Renew Sustain Energ Rev. 2017; 76: $577-$ 607.

3. Choton K. Das, Octavian Bass, Ganesh Kothapalli, Thair S. Mahmoud, Daryoush Habibi. Overview of energy storage systems in distribution networks: Placement, sizing, operation, and power quality. Renew. Sustain Energ Rev. 2018; 91: 1205-1230.

4. Jalali A, Aldeen M. Risk-based stochastic allocation of ESS to ensure voltage stability margin for 
distribution systems. IEEE Trans Power Syst. 2019; 34(2): 1264-1277.

5. Choton K. Das, Octavian Bass, Ganesh Kothapalli, Thair S. Mahmoud, Daryoush Habibi. Optimal placement of distributed energy storage systems in distribution networks using artificial bee colony algorithm. Appl Energ. 2018; 232: 212-228.

6. Tsikalakis A, Hatzargyriou N. Centralized control for optimizing microgrids operation. IEEE Trans Energ Convers. 2008; 23(1): 241-248.

7. Hoke A, Brissette A, Chandler S, Pratt A, Maksimovic D. Lookahead economic dispatch of microgrids with energy storage, using linear programming. IEEE Conf Technol Sustain. 2013.

8. Lively M. Creating a MicroGrid market: using a frequency driven pricing curve to dispatch load and embedded distributed generation and to charge and pay for participation. Energ Centr. 2013.

9. Ali Ehsana, Qiang Yang. State-of-the-art techniques for modelling of uncertainties in active distribution network planning: A review. Appl Energ. 2019; 239: 1509-1523.

10. Mohammad Rasol Jannesar, Alireza Sedighi, Mehdi Savaghebi, Josep M. Guerrero. Optimal placement, sizing, and daily charge/discharge of battery energy storage in low voltage distribution network with high photovoltaic penetration. Appl Energ. 2018; 226: $957-$ 966.

11. Hossein Fallahzadeh-Abarghouei, Saeed Hasanvand, Ahmad Nikoobakht, Meysam Doostizadeh. Decentralized and hierarchical voltage management of renewable energy resources in distribution smart grid. Int J Elect Power Energ Syst. 2018; 100: 117-128.

12. Hatziargyriou ND, Dimeas AL, Tsikalakis AG, Pecas Lopes JA, Kariniotakis G, Oyarzabal J. Management of MicroGrids in market environment. IEEE Int Conf Fut Power Syst. 2005.

13. Georgios C. Kryonidis, Charis S. Demoulias, Grigoris K. Papagiannis. A new voltage control scheme for active medium-voltage (MV) networks. Elect Power Syst Res. 2019; 169: 53-64.

14. Yasmeen A, Javaid N, Zahoor S, Iftikhar $H$. Optimal energy management in microgrids using metaheuristic technique. Int Conf Emerg Internet Data Web Technol. Adv Internet Data Web Technol. 2018; 303-314.

15. José Iriaa, Miguel Heleno, Gonçalo Cardoso. Optimal sizing and placement of energy storage systems and on-load tap changer transformers in distribution networks. ApplEnerg. 2019; 250: 1147-1157. 16. Ling Ai Wonga, Vigna K. Ramachandaramurthy, Phil Taylora, JB Ekanayaked, Sara L. Walker, Sanjeevikumar Padmanabane, Review on the optimal placement, sizing and control of an energy storage system in the distribution network. J Energ Stor. 2019; 21: 489-504.

17. Sajad Mahdavi, Reza Hemmati, Mehdi Ahmadi Jirdehi. Two-level planning for coordination of energy storage systems and wind-solardiesel units in active distribution network. Energ. 2018; 151: 954-965.

18. Shu Wang, Fengji Luo, ZhaoYang Donga, Gianluca Ranzi. Joint planning of active distribution networks considering renewable power uncertainty. Int $J$ Electr Power Energ Syst. 2019; 110: 696-704.

19. Plecas M, Xu H, Kockar I. Integration of energy storage to improve utilization of distribution networks with active network management shemes. CIRED, Open Access Proc J. 2017; 1: 1845-1848.

20. Yongxi Zhang, Shuyun Ren, Zhao Yang Dong, Yan $\mathrm{Xu}$, Ke Meng, Yu Zheng. Optimal placement of battery energy storage in distribution networks considering conservation voltage reduction and stochastic load composition. IET Gener Transm Distrib. 2017; 11(15): 3862-3870.

21. Maleki A, Askarzadeh A. Optimal sizing of a $\mathrm{PV} /$ wind/diesel system with battery storage for electrification to an off-grid remote region: A case study of Rafsanjan, Iran. Sustain Energ Technol Assess. 2014; 7: 147-153.

22. Jijian Liana, Yusheng Zhanga, Chao Maa, Yang Yanga, Evance Chaima. A review on recent sizing methodologies of hybrid renewable energy systems. Energ Convers Manag. 2019; 199.

23. Kumar M, Nallagownden P, Elamvazuth I. Optimal placement and sizing of renewable distributed generations and capacitor banks into radial distribution systems. Energ. 2017; 10: 1-25.

24. Davide Fioriti, Davide Poli. A novel stochastic method to dispatch microgrids using Monte Carlo scenarios. Electr Power Syst Res. 2019; 175.

25. Mohsin M, Rao KVS. Estimation of weibull distribution parameters and wind power density for wind farm site at Akal at Jaisalmer in Rajasthan. International Innovative Applications of Computational Intelligence on Power, Energy and Controls with their Impact on Humanity (CIPECH), 2018; 14-19.

26. Chauhan A, Saini RP. Statistical analysis of wind speed data using Weibull distribution parameters. Int Conf Non-Convent Energ. (ICONCE 2014), 2014; 160163.

27. Sheng W, Ke-Yan Liu, Liu Y, Meng X, Li Y. Optimal placement and sizing of distributed generation via an improved nondominated sorting genetic algorithm II. IEEE Trans Power Deliv. 2015; 30(2): 569578. 


\section{SJIS}

Copyright: (C) 2021 The Author(s); This is an open-access article distributed under the terms of the Creative Commons Attribution License (http://creativecommons.org/licenses/by/4.0), which permits unrestricted use, distribution, and reproduction in any medium, provided the original work is properly cited.

Citation: Kalantari M. Optimal Design and Scheduling of Active Distribution Network with Penetration of PV/Wind/BESS Energy Systems Considering the Load Side Management. SJIS, 2021; 3(3): 1-13.

https://doi.org/10.47176/sjis.3.3.1 